\title{
LA BIODIVERSITÉ TROPICALE
}

\author{
JEAN DEMANGEOT ${ }^{1}$
}

Amical hommage

à Suzanne Daveau-Ribeiro,

tropicaliste exemplaire

Résumé - La biodiversité, c'est à dire la variété des espèces vivantes est considérable dans les régions intertropicales. On l'explique par la grande diversité des niches écologiques, génératrice d'adaptations et de spéciations nombreuses; par la grande superficie des régions intertropicales (29\% des terres émergées); par l'extrême ancienneté de l'émersion des socles géologiques (cordillères exceptées naturellement).

Mots-clés: Pays tropicaux, biodiversité tropicale, niches écologiques, surface continentale, émersions géologiques, adaptation, spéciation.

Resumo - A BIODIVERSIDADE TROPICAL - A biodiversidade, isto é a variedades das espécies, é considerável nas regiões intertropicais. A explicação deste facto reside na grande diversidade de nichos ecológicos, geradora de numerosas adaptações e especiações, na grande área das regiões intertropicais (29\% das terras emersas) e na antiguidade da emersão do soco geológico (à excepção das cadeias montanhosas).

Palavras-chave: Países tropicais, biodiversidade tropical, nichos ecológicos, superfície continental, emersão geológica, adaptação, especiação.

La biodiversité est à la mode ${ }^{2}$ et c'est tant mieux. N'est-il pas affligeant de ne trouver que 10 variétés de pommes et 4 variétés de prunes au marché?

Or on sait que les régions intertropicales sont les plus riches en espèces animales et végétales du monde. En voici quelques exemples (il faut toujours tenir compte des surfaces: car le nombre des espèces croît comme le logarithme de la surface considerée):

- Richesse floristique de l'Afrique sur $10000 \mathrm{~km}^{2}: 1000$ à 1200 espèces en zone sahélienne, 2000 dans la Forêt dense d'Afrique occidentale et centrale, 3200 à 3800

1 Professeur honoraire des Universités, Membre de l'Académie des Sciences d'Outre-Mer, Paris. 6, Rue de Chézy. F - 92200 Neuilly-sur-Seine. France.

2 LeVeque, C., (1997) La biodiversité. in QUE SAIS-JE?. PUF,3, (166), Paris: 128 p. 
dans la région des Grands lacs, 5400 à Madagascar (mais 1000 environ pour les plaines européennes) ${ }^{3}$;

- Palmiers: 320 taxons de Palmiers utiles dans le monde, appartenant à 243 genres et susceptibles de 391 usages pratiques: or les $4 / 5$ de ces taxons sont tropicaux ${ }^{4}$;

- Richesse floristique d'une forêt pluviale de Guyana sur 1,5 ha: 432 espèces de plus de $10 \mathrm{~cm}$ de diamètre, 232 espèces de plus de $20 \mathrm{~cm}, 60$ espèces de plus de 41 $\mathrm{cm}^{5}$;

- Faune des savanes de Lamto, Côte d'Ivoire: 314 Vertébrés dont 230 Oiseaux; 1 mollusque (?); 11 Oligochètes; 194 Araignées; 2543 Insectes (?) dont 800 Coléoptères et 500 Diptères 6 ;

- Poissons capables de respirer à l'air libre dans les eaux de mer de Panama: 40 espèces, appartenant à 6 familles 7 .

Au regard de ces chiffres notons que la France, par exemple, sur $550000 \mathrm{~km}^{2}$, ne possède que 89 espèces de mammifères ${ }^{8}$ dont 8 ongulés, etc.

Quelles sont les causes de cette remarquable variété de la vie dans les Intertropiques? La diversité des milieux habitables, répond l'écologiste; la grande surface des terres émergées, répond le géographe; la longue filiation des taxons, répond le paléontologiste. Bref c'est un problème d'évolution ${ }^{9}$.

\section{1 - DIVERSITÉ DES NICHES ÉCOLOGIQUES}

Qui dit biodiversité dit spéciation (c'est-à-dire naissance des espèces) et qui dit spéciation dit adaptation au milieu. Or cette adaptation implique d'abord la sélection naturelle, c'est-à-dire la disparition des plus faibles (jeunes, malades, vieux). Puis une plasticité génétique, condition sine qua non parfois appelée pré-adaptation, puis une adaptation autant génétique que morphologique, la première étant le gage d'une interfécondité. Une preuve bien connue de l'influence du milieu est la convergence des formes chez des espèces différentes et aussi le mimétisme: mais la plupart des mécanismes nous échappent encore. L'adaptation est donc liée à une modification du milieu donc à l'apparition d'une situation nouvelle: modification climatique (on parle alors d'anagenèse), apparition d'un obstacle géographique qui coupe le milieu initial en deux ou trois (par exemple, surrection

3 OzENDA, P. (1964) Bíogéographie végétale. Doin, Paris: 374 p.

4 BALICK, M.; H. BECK (1990) Useful Palms of the World, a Synoptic Bibliography. Columbia Univ. Press, New York: 724 p.

5 Richards, P. (1976) The Tropical Rain Forest. Cambridge Unív. Press: 450 p.

6 Lamotte, M.(1994) Théorie actuelle de l'évolution. Hachette, Paris: 444 p.

7 Graham, J. (1982) in Evolucion en los Tropicos. Smithsonian Tropical Research Institute, Panama: $292 \mathrm{p}$.

8 Saint Girons, M. Ch. (1989) Les mammifères en France. Sang de la Terre, Paris: 249 p.

9 J'ai beaucoup utilisé, pour écrire ces lignes, CUENOT (1932) La genèse des espèces animales. Alcan, Paris: 822 p.; Delsol, M. (1989) Cause, loi, hasard en biologie. Vrin, Paris: 241 p.; ForD, E. (1972) Génétique écologique. Gauthier-Villars, Paris: 448 p.; LAmotTe, M. (1994) Théorie actuelle de l'évolution. Hachette, Paris: 444 p.; et les divers articles de Rostand, J. et A. TETRY (1965) Biologie. Gallimard, Encyclopédie de la Pléiade, Paris: 2036 p. 
de l'isthme d'Amérique centrale qui isole les eaux du Pacifique des eaux de l'Atlantique). Il s'agit alors d'une «cladogenèse». Dans un cas comme dans l'autre, l'adaptation acquise, le retour en arrière est impossible (loi de Dollo).

Pour ce qui nous concerne il est évident que les régions tropicales sont constituées de milieux extrêmement variés. Et encore je ne considère ici que les plaines et plateaux des vieilles plates-formes, excluant du discours les Cordillères et jeunes chaînes qui sont un monde complètement différent. Le facteur commun de tous les pays tropicaux non désertiques est la chaleur: plus ou moins humide, plus ou moins continue. Mais l'exposition au soleil (car même hors des montagnes les basses latitudes connaissent des problèmes d'insolation), l'exposition aux vents plus ou moins secs (harmattan), aux coulées froides («friagem» d'Amazonie), à la pluie, les détails de l'hydrologie de surface (biefs d'eau calme, rapides, marécages, águas limpas, águas pretas, águas brancas, rives inondées ou non, etc.), les fantaisies incontrôlables de l'hydrologie souterraine, les innombrables nuances des formations végétales (espèces forestières, stratification, chablis, repousses), les variétés de surfaces dures, cuirasses à divers niveaux et de diverses compositions, variété géologique des dômes rocheux et des pains de sucre, glissements de terrain, intervention des termites, des éléphants, des jacinthes d'eau, brûlis anthropiques plurimillénaires, etc., tout, absolument tout concourt à créer un immense assortiment de niches écologiques. Donc les nécessités d'adaptation, donc les occasions de spéciation. Ici, plus qu'ailleurs peut-être, la biodiversité est clairement liée à l'écodiversité.

On n'a alors que l'embarras du choix pour citer les cas d'adaptation. Dans le règne végétal (voir travaux de SCHNELL) tel genre, la Landolphia par exemple, est tantôt buissonnant tantôt lianescent, selon la lumière. La forme des feuilles en forêt dense, allongée, bien étalée, facilite l'évaporation; la pointe en bec verseur facilite l'écoulement de l'eau. Inversement la convergence morphologique des Cactées américaines et des Euphorbiacées africaines (surface vernissée, épines) est une admirable démonstration de l'influence d'une sécheresse modérée. On trouve pareille convergence dans le règne animal. Dans une planche illustrée saisissante LAMOTTE compare le comportement ou la forme d'espèces différentes (fig. 1). La couleur vive et filtrante de nombreux insectes, Papillons surtout, les longues pattes et les longues oreilles des quadrupèdes (loi d'Allen), la grande taille des animaux à sang froid (l'inverse de la loi de Bergmann) tels que le boa, l'anaconda, le Varan de Komodo, la couleur des animaux plus claire dans le Sahel que dans le tropical humide (loi de Gloger), autant d'adaptations au polymorphisme de la nature tropicale. Enfin, beaucoup d'adaptations, beaucoup d'espèces, donc beaucoup de concurrence: le mimétisme avec les espèces mieux défendues, chez les Papillons par exemple, ou le camouflage pour se fondre dans le décor (couleur verte des grenouilles, ocelles du jaguar, rayures du zèbre) sont l'expression de ces multiples ruses naturelles destinées à assurer la survie. 

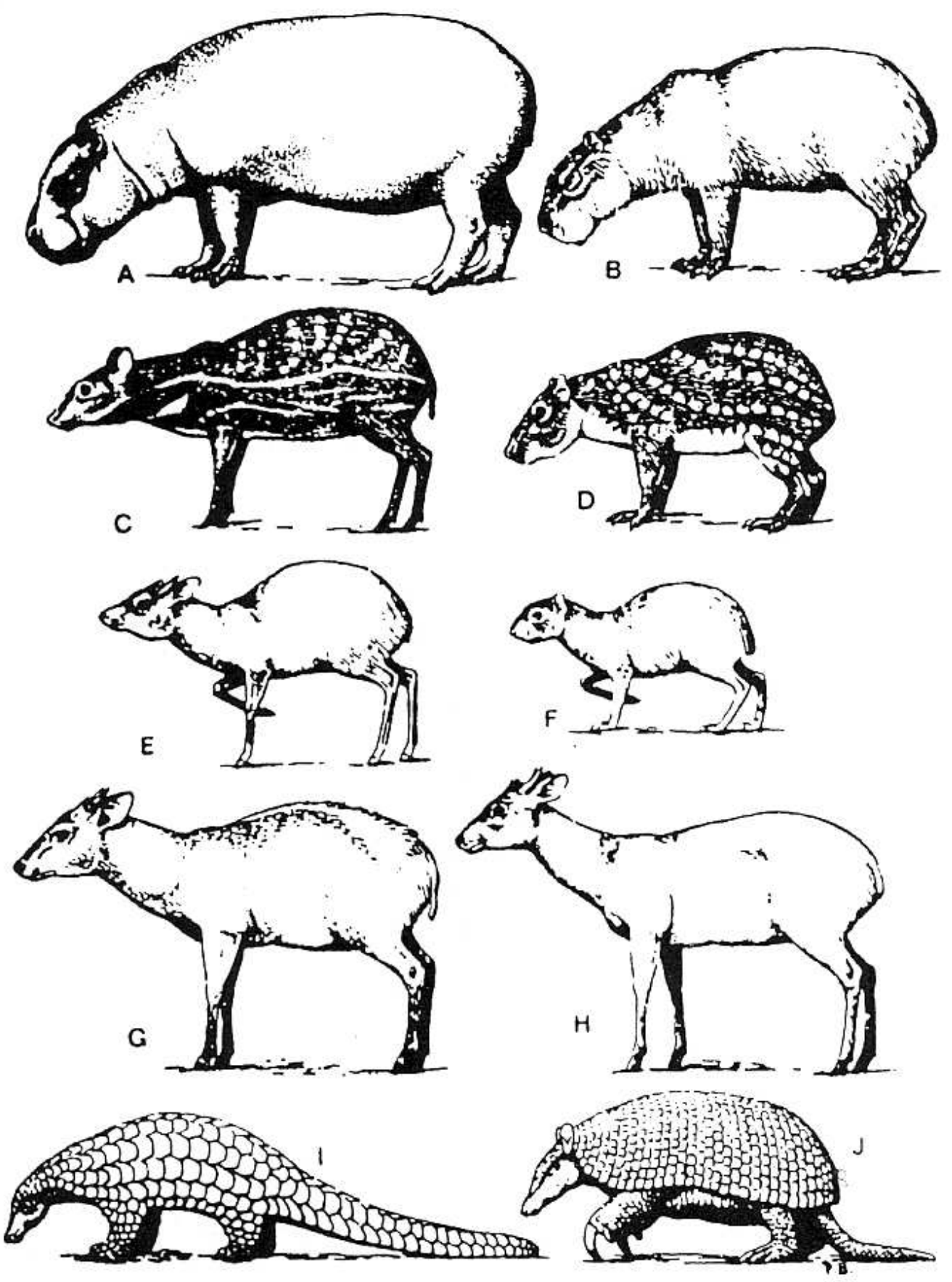

Fig. 1 - Convergence morphologique entre les Mammifères forestiers africains (à gauche) et d'Amérique du Sud (à droite). A: Hippopotame nain Choeropsis liberiensis; B: Capybara Hydrochaerus hydrochaeris, le plus grand Rongeur actuel, pouvant peser $50 \mathrm{~kg}$; C: Chevrotain africain Hyemoschus aquaticus; D: Rongeur Paca Cuniculus paca; E: Antilope royale; F: Rongeur Agouti du genre Dasyproctus; G: Antilope forestière Cephalophus sylvicultor; H: Cerf forestier Mazama gouazoubira; I: Pangolin terrestre; J: Tatou géant (d'après Dubost, 1968 et Bourlière, 1973, in Lamotte, 1994, p. 68).

Fig. 1 - Convergência morfológica entre os mamíferos florestais africanos (à esquerda) e da América do Sul (à direita). 
Les pays très froids nous fournissent au contraire l'exemple d'une simplification, voire d'une uniformisation des milieux. En effet si les reliefs hyperboréens sont tout aussi variés que ceux des pays chauds, leurs effets sont annulés par la toute puissance du froid. Pendant les 8 à 9 mois d'hiver l'air à -15 ou $-30^{\circ} \mathrm{C}$ baigne indifféremment les creux et les bosses du paysage, quelle que soit l'orientation (il fait nuit très longtemps chaque jour) et il impose son uniformité léthale à l'ensemble du biota. En été la vie reprend, avec vigueur, certes, mais pour très peu de mois. D'autre part, l'action dirimante du froid atmosphérique d'hiver est partiellement relayée par celle de la glace de sol (permafrost), qui limite l'enfoncement des racines et la vie souterraine, y compris celle des microorganismes de la pédogenèse. Il y a des niches écologiques contrastées, bien sûr, mais elles ne sont fonctionnelles que quelques mois seulement. Si l'adaptation du biota est qualitativement remarquable (rythme des floraisons, nanisme végétal, fourrure et grande taille des mammifères selon la loi de Bergmann), elle est insuffisante pour induire une spéciation importante et diversifiée. Une preuve indirecte de cette affirmation est la variété et le foisonnement de la vie dans les eaux marines arctiques qui, elles, sont protégées des froids extrêmes par la glace.

\section{2 - L'EXTENSION TERRITORIALE}

Plus une surface continentale est grande plus elle a de chances statistiques d'être écologiquement variée, ne serait-ce que par sa topographie, son développement en latitude, facteur de variation thermique, son degré de continentalité, facteur de variation thermique et pluviométrique. OZENDA rappelle que «l'on constate que le nombre d'espèces (floristiques) croît comme le logarithme de la surface prise en considération» ${ }^{10}$ : on ne peut donc pas comparer le nombre des espèces de Ceylan et celui de l'Inde entière, par exemple, sans apporter une correction due à la surface territoriale. Or les pays tropicaux que je considère ici, c'est-à-dire les parties tropicales des continents, cordillères exclues, sont très étendus, et par grands ensembles continus. Qu'on en juge plutôt (tableau I).

Ces chiffres résultent d'un planimétrage, mais les problèmes d'échelle, de projection, de limites (les écotones!) les rendent évidemment inexacts: on ne retiendra que les ordres de grandeur. A savoir que les milieux tropicaux, cordillères exclues $\left(4,8 \mathrm{M} \mathrm{km}^{2}\right)$, mesurent à peu près $43 \mathrm{M} \mathrm{km}^{2}$; qu'ils sont répartis sur deux continents d'un seul tenant, l'Amérique $\left(15,8 \mathrm{M} \mathrm{km}^{2}\right)$ et l'Afrique $\left(17,3 \mathrm{M} \mathrm{km}^{2}\right)$; sur un continent fortement insulaire, l'Asie $\left(6,7 \mathrm{M} \mathrm{km}^{2}\right)$; et sur un continent majoritairement insulaire, l'Océanie $\left(3,2 \mathrm{M} \mathrm{km}^{2}\right)$. Naturellement, dans une étude plus détaillée, il faudrait prendre en compte les liaisons latérales: Afrique fortement isolée, Amérique centrale à éclipses, Asie insulaire également incertaine au cours des temps, mais tout de même généralement reliée à l'Eurasie, etc.

10 OZENDA, loc. cit. 
Tableau I: Surface des milieux continentaux tropicaux ${ }^{11}$ Quatro I: Área dos ambientes continentais tropicais

\begin{tabular}{|c|c|c|}
\hline Milieux tropicaux & en $\mathrm{M} \mathrm{km}^{2}$ & en \% \\
\hline $\begin{array}{l}\text { (forêts et savanes confondues) } \\
\text { dont: ombrophiles } \\
\text { mésophiles } \\
\text { tropophiles } \\
\text { sahéliens }\end{array}$ & $\begin{array}{c}6,8 \\
12,8 \\
13,8 \\
10,6 \\
43,6\end{array}$ & $\begin{array}{c}4,5 \\
8,2 \\
9,4 \\
7,0 \\
29,1\end{array}$ \\
\hline
\end{tabular}

\begin{tabular}{|c|c|c|}
\hline Milieux froids (pour comparaison) & & \\
\hline $\begin{array}{l}\text { circumpolaires } \\
\text { boréaux }\end{array}$ & $\begin{array}{c}4,1 \\
15,5 \\
19,6\end{array}$ & $\begin{array}{c}2,8 \\
10,3 \\
13,1\end{array}$ \\
\hline
\end{tabular}

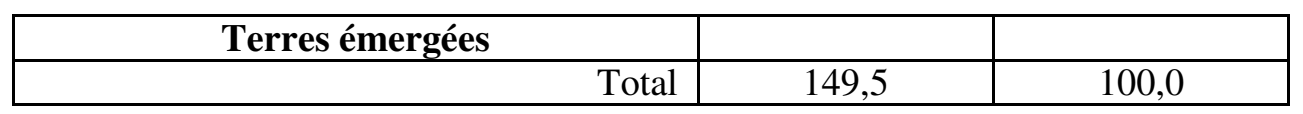

Source: J. Demangeot, 1996.

Le corollaire de cette importance des grandes surfaces pour la richesse en espèces est le cas des isolats de taille moyenne ou petite, îles, grands lacs, massifs montagneux, etc. Là, les taxons ont certes tendance à se spécialiser: et c'est l'endémisme. Mais en même temps les autres espèces s'étiolent et disparaissent. C'est tout le contraire de la prolifération spécifique.

\section{3 - LE FACTEUR «TEMPS»}

Résultant d'une évolution complexe, la spéciation exige de longs intervalles de temps. Certes, dans les expériences de laboratoire, on travaille par commodité sur des espèces qui se multiplient rapidement: la Drosophile, par exemple, qui vit une quinzaine de jours. Mais en paléontologie on est contraint d'envisager le destin de toutes les familles de tous les règnes, et l'unité de temps n'est plus l'année mais le million d'années. Les chiffres varient beaucoup selon les espèces. Selon DELSOL et LAMOTTE, il faut de 300 millénaires à 10 millions d'années pour qu'une nouvelle espèce apparaisse. Et il semble bien, d'après la bibliographie, que les spéciations marines soient plus lentes que les spéciations continentales.

Or il est bien connu que les climats intertropicaux s'exercent (hors cordillères) sur de très vieilles plates-formes géologiques très longtemps émergées à l'air libre. On s'excuse de rappeler que la Pangée précambrienne s'est progressivement dislo-

11 Demangeot, J. (1996) Les milieux naturels du globe. 60 éd., A., Colin, Paris: 337 p.; et article in Aspects du monde tropical et asiatique, hommage à Jean Delvert, (1991), Presses de l'Université Paris-Sorbonne, Paris: 377 p. 
quée en deux grands ensembles fondamentaux, la Gondwanie et la Laurasie; que ces ensembles se sont eux-mêmes subdivisés en plaques distinctes qui ont «vogué» isolément sous la contrainte des fonds océaniques en expansion; que, pendant leurs déplacements, ces plaques sont presque tout le temps restées à l'air libre: les transgressions marines y ont été plutôt rares, par exemple la mer crétacée en Afrique de l'ouest; que ces plaques ont été empâtées par leurs propres débris; que, par conséquent, chaque plaque comporte un socle généralement précambrien et une couverture discordante plus récente; que ces couvertures nous renseignent sur les climats passés; que nous savons donc qu'au début les climats ont été généralement secs et froids (il y a même eu des glaciations, par exemple la glaciation carbonifère); mais qu'ensuite les continents gondwaniens se sont trouvés sous climat chaud, tantôt sec, tantôt humide; et que, par conséquent (last but not least), la plupart des taxons tropicaux actuels sont issus d'un passé généralement tropical de 80 millions d'années... Belle durée qui a permis la biodiversité dont il est question.

De nos jours on retrouve donc:

a) des espèces gondwaniennes extraordinairement anciennes, tels les Dipneustes, poissons pulmonés présents en Amérique du sud, en Afrique, en Australie,

b) des groupes nés sur tel continent tropical mais absents sur tel autre: par exemple les Singes Platyrhiniens en Amérique et les Singes Catarhiniens dans l'Ancien monde,

c) des groupes nés ailleurs puis immigrés en terre tropicale: par exemple l'arrivée de Mammifères prédateurs du Mexique en Amérique du sud par l'isthme de Panama (dommageables, d'ailleurs, aux taxons méridionaux).

Grâce à cette histoire géologique, les taxons tropicaux sont donc en général extrêmement anciens et diversifiés.

On pourrait objecter que la Laurasie (Amérique du nord + Ancien monde non tropical, en gros) a été très longtemps émergée et que ses boucliers géologiques se prêtaient également bien à la spéciation: ce serait oublier que les effets biologiques de cette longue émersion ont été gommés, voire rabotés, par les grands glaciers quaternaires pendant 3 millions d'années et leur auréole de périglaciaire. Cela a été une grande chance pour les Intertropiques de ne pas connaître pareille catastrophe.

J'espère avoir montré que la biodiversité tropicale résulte bien de la combinaison des adaptations du biota à de multiples niches écologiques, sur de vastes territoires et pendant des durées considérables. Or cette diversité est précieuse. Sans verser dans un écologisme béat il faut bien avoir conscience qu'elle correspond non seulement à une certaine éthique humaniste mais aussi qu'elle a des implications pratiques concrètes. $\mathrm{Ce}$ n'est pas pour rien que la firme pharmaceutique Merck a loué à l'Etat $25 \%$ du Costa Rica: il est clair que les deux parties ont intérêt à ce que la nature ne s'appauvrisse pas ${ }^{12}$.

12 LEVEQUE, op. cit. 